Published in final edited form as:

Urology. 2018 October ; 120: 109-113. doi:10.1016/j.urology.2018.06.036.

\title{
The Impact of Microsurgical Repair of Subclinical and Clinical Varicoceles on Total Motile Sperm Count: Is There a Difference?
}

\author{
Nannan Thirumavalavan ${ }^{1}$, Jason M ScovelI ${ }^{1,2}$, Adithya Balasubramanian ${ }^{1}$, Taylor P Kohn ${ }^{1}$, \\ Byung Ji ${ }^{1}$, Asad Hasan ${ }^{1}$, Alexander W Pastuszak ${ }^{1}$, and Larry I Lipshultz ${ }^{1}$ \\ ${ }^{1}$ Department of Urology, Baylor College of Medicine, Houston, TX \\ ${ }^{2}$ Translational Biology and Molecular Medicine, Baylor College of Medicine, Houston, Texas
}

\begin{abstract}
Objectives: To determine if subclinical varicocele repair produces similar results to palpable varicocele repair.

Methods: Retrospective review was performed on 190 infertile men who underwent a microsurgical varicocele repair by two surgeons from 2009 to 2017. Improvement in total motile sperm count (TMC) that enables men limited to in vitro fertilization (IVF) or intrauterine insemination (IUI) to undergo IUI or natural conception ('upgrade') is clinically meaningful. Using TMC, men were grouped into three pre- and postoperative categories: IVF, (TMC $<5$ million), IUI (TMC 5-9 million), or natural pregnancy (TMC $>9$ million). Changes in category after varicocele repair were assessed. We compared the proportion of men in each category with clinical varicoceles to those with subclinical varicoceles.
\end{abstract}

Results: Men with clinical and subclinical varicoceles had improvements in TMC after surgery (change in TMC of $9.3 \pm 19.5,7.7 \pm 22.6$ million, $\mathrm{p}<0.001$ for both, respectively). There was no difference in TMC improvement between men with clinical and subclinical varicoceles $(\mathrm{p}=0.66)$. Of men initially limited to IVF, $11 \%$ improved to IUI, and $38 \%$ to natural pregnancy. Of patients starting in IUI category, $22 \%$ transitioned to natural pregnancy category. No difference exists in the proportion of men who "upgraded" between palpable or subclinical varicoceles.

Conclusions: Men with subclinical varicoceles have similar, clinically meaningful improvement in TMC after varicocele repair compared to men with palpable varicoceles.

\section{Keywords}

varicocele; subclinical; varicocelectomy; varicocele repair; ultrasound; infertility

\footnotetext{
*Address for Correspondence: Nannan Thirumavalavan, 1 Baylor Plaza, Alkek N730, Houston, TX 77030, (713) 798-6266, nannan@bu.edu.

Publisher's Disclaimer: This is a PDF file of an unedited manuscript that has been accepted for publication. As a service to our customers we are providing this early version of the manuscript. The manuscript will undergo copyediting, typesetting, and review of the resulting proof before it is published in its final citable form. Please note that during the production process errors may be discovered which could affect the content, and all legal disclaimers that apply to the journal pertain.
} 


\section{Introduction}

Though varicoceles have long been implicated as a factor in male infertility, the extent of their effect remains unknown. Varicocele repair improves semen parameters and live birth outcomes in some men with infertility ${ }^{1,2}$, and can improve outcomes together with the use of assisted reproductive technology (ART). ${ }^{2,3}$ It is currently unclear which men will benefit from varicocele repair, as approximately $15 \%$ of all men have varicoceles. ${ }^{4-6}$ Not all men with a varicocele with experience infertility, and not all infertile men with a varicocele will have improved fertility after surgery. Since most studies have focused on clinically palpable varicoceles, current guidelines support varicocele repair only for palpable varicoceles. ${ }^{7}$

However, some evidence suggests that repair of subclinical varicoceles, which are not palpable but are detected by ultrasound (US), may benefit men with infertility. ${ }^{8} \mathrm{~A}$ recent meta-analysis of seven well designed trials concluded that more evidence is needed to make final recommendations. ${ }^{9}$ Given this lack of data regarding subclinical varicoceles, we sought to determine the impact on male fertility, as assessed by total motile sperm count (TMC) after repair of subclinical varicoceles.

Evaluation of live birth is the gold standard to assess the efficacy of fertility treatment. However, obtaining live birth outcomes is challenging, and an improvement in semen parameters is often used as a surrogate in determining the efficacy of varicocele repair. It is difficult to interpret the clinical impact that an increase in TMC has on fertility outcomes. To address this limitation, the concept of "upgrade" in total motile count has been used. ${ }^{10,11}$ An increase in TMC that enables a man initially limited to in vitro fertilization (IVF) or intrauterine insemination (IUI) due to low sperm density, to now undergo IUI or natural conception ('upgrade') is a clinically meaningful change. ${ }^{11}$ Using this framework, we determined the proportion of men who 'upgrade' after varicocele repair with a subclinical varicocele (identified by ultrasound) and if this differs from the rate of 'upgrade' in men with palpable varicoceles.

\section{Methods}

Patients who underwent microsurgical varicocele repair by one of two surgeons (LIL and AWP) between 2009 and 2017 were identified using billing data. An IRB approved retrospective chart review was performed on all patients who underwent varicocele repair for infertility. We included 190 infertile male patients who had both preoperative and postoperative semen analyses performed in a single andrology laboratory. Baseline characteristics included age, body mass index (BMI), smoking status, primary vs. secondary infertility, and duration of infertility. Available pregnancy data recorded in the chart was documented.

A standard new male infertility evaluation at our clinic includes a thorough history and physical, hormonal parameters, one semen analysis, and a scrotal ultrasound. Men presenting for infertility at our center are examined in the standing position. The room temperature is usually adequately warm, and all patients are asked to Valsalva during when they are being examined for varicoceles. The attending's exam is documented separately 
from trainee exams and was used for our analysis. Scrotal US is performed by a dedicated ultrasound technician in supine, and subsequently standing position. A varicocele is diagnosed if a venous diameter is greater than $3 \mathrm{~mm}$, and retrograde flow is confirmed. If the patient presenting with infertility has a varicocele, either palpable or subclinical, and one abnormality on semen analysis, a varicocele repair is offered.

We preferentially use the inguinal approach for varicocele ligation unless contraindications such as previous inguinal surgeries are present. In these cases, a sub-inguinal approach is used. A five to six cm incision is made superior and laterally to the external inguinal ring. The external oblique fascia is identified and incised, and the cord is delivered onto the field. The testicle is not routinely delivered. The vas deferens and associated vessels are isolated under the microscope. Direct microscopic visualization and micro-tip Doppler ultrasound (VTI Instruments) are used to identify the arteries in the cord. The veins are then isolated and tied off using 3-0 and 2-0 silk ties. Prior to replacing the cord into the inguinal canal, arterial flow is confirmed using the Doppler. The incision is then closed. Postoperatively, patients are seen at 16 weeks for an office visit and semen analysis, with subsequent semen analyses repeated every 3 months thereafter on average. Comparisons were made using either the 1 year postoperative semen analysis or when unavailable, the latest available analysis.

Based on sperm density and its correlation with the appropriate ART, men were grouped into three pre and postoperative categories using TMC as follows: IVF, ( $<5$ million TMC), IUI (5 -9 million TMC), and natural pregnancy ( $>9$ million TMC). ${ }^{11}$ The changes in category after surgery were assessed. We compared the proportion of men in each pre and post-operative category who had palpable varicoceles to those who had subclinical varicoceles and used Fisher's exact test to compare groups. Of note, if patients had a palpable varicocele on one side and a subclinical on the other side, they were classified as palpable for the analyses. A paired Student's t-test was used to compare differences in baseline parameters, and pre- and postoperative TMC. All analyses were performed using SPSS v22 (IBM, NY). To determine the study's power, we performed a Post-hoc power analysis to detect a 10 million TMC between groups, which yielded a power of 0.835 (G*Power 3.1).

\section{Results}

The mean \pm SD age of men undergoing varicocele repair was $35.1 \pm 6.1$ years old (range 24-56), and the mean partner age was $31 \pm 4.1$ years old (range 22-42). Of all men, $88 \%$ presented with primary infertility, and $12 \%$ with secondary infertility. Couples had been attempting to initiate pregnancy for 21 months on average. Mean \pm SD BMI was 29.5 \pm 7.3 and $9.6 \%$ of the cohort were smokers. Bilateral varicocele repair was performed on $74 \%$ of men. No differences in baseline semen parameters was observed between men with palpable and subclinical varicoceles (Table 1).

For all men who underwent a varicocele repair, the mean \pm SD improvement in TMC was 8.9 \pm 20.2 million $(\mathrm{p}<0.00001)$. Of men who were preoperatively characterized as candidates for IVF, $49 \%$ remained in this group after surgery, 13\% upgraded to the IUI category, and $38 \%$ to the natural pregnancy category (Table 2). Of men who were preoperatively categorized as 
IUI candidates, $13 \%$ were downgraded to the IVF category, $13 \%$ remained in the IUI category, and $73 \%$ were upgraded to the natural pregnancy category. Of men who were preoperatively categorized as candidates for natural pregnancy, $11 \%$ were downgraded to the IVF category and $9 \%$ to the IUI category, with $80 \%$ remaining in the natural pregnancy category. Of the 85 men who were in the natural pregnancy category preoperatively, the mean \pm SD improvement in TMC was $8.1 \pm 26.4$ million, with 50 of the $85(59 \%)$ having improved TMC.

Both men with palpable and subclinical varicoceles had significant improvements in TMC after repair. Men with palpable varicoceles had an increase in TMC of $9.3 \pm 19.5$ million sperm ( $\mathrm{p}<0.001$ ), and men with subclinical varicoceles improved by $7.7 \pm 22.6$ million $(\mathrm{p}<0.001)$. No differences in TMC improvement between men with palpable and subclinical varicoceles were observed $(\mathrm{p}=0.66)$. Rates of upgrade between fertility category were similar between men with palpable and subclinical varicoceles (Table 3).

Given that some patients had a palpable varicocele on one side and non-palpable on the other, secondary analysis was performed to address this potential confounder. When comparing $(n=58)$ patients undergoing bilateral varicocelectomy for bilaterally palpable varicoceles to patients with bilaterally nonpalpable varicoceles who underwent bilateral repair $(n=31)$, there was still no difference in improvement in total motile counts $(\mathrm{p}=0.75)$. In general, there was no difference in improvements in total motile counts between patients undergoing bilateral and unilateral repair $(10.49+/-22.4$ vs 8.96+/- 19.8 million TMC, $\mathrm{p}=0.68)$.

\section{Discussion}

Our results support evidence that advocates for evaluation and repair of subclinical varicoceles. Previous work has shown that repairing a small but palpable varicocele contralateral to a large palpable varicocele conveys significant benefit to repairing only the larger side. ${ }^{12}$ In a series comparing men who underwent varicocele repair for either subclinical $(n=73)$ or palpable varicoceles $(n=77)$, Pierik et al. found that although total sperm counts increased more in men with clinical varicoceles, motility increased more in men with subclinical varicoceles after repair. ${ }^{8}$ Steckel et al. also found that in palpable varicoceles, size was associated with improvement after repair. ${ }^{13}$ Cantoro et al. compared 218 infertile men who underwent percutaneous embolization of subclinical left sided varicoceles to 119 infertile control men with varicoceles who did not have intervention. ${ }^{14}$ The treatment group showed significant improvements in mean sperm concentration and total motility. Most notably, after approximately 3 years of follow up, the intervention group achieved a higher pregnancy rate than the control group ( $46.3 \%$ vs $11.8 \% ; \mathrm{p}=0.01$ ). Seo et al. retrospectively reviewed 143 men with subclinical left sided varicoceles, of which 25 underwent microsurgical varicocele repair, 93 underwent medical management with Lcarnitine, and 25 were controls. ${ }^{15}$ Sperm counts improved in the surgery group and after 1 to 2 years of follow-up, and the pregnancy rate was significantly higher in the surgery group compared with controls (60\% vs $18.7 \%$; $\mathrm{p}=0.03$ ). 
Several small prospective studies have evaluated the impact of repairing subclinical varicoceles on sperm counts and fertility. A study by Unal et al. randomized 42 nonazoospermic patients ( 21 in each arm) to either clomiphene alone or surgery with high ligation of the internal spermatic vein. The natural pregnancy rate was $12.5 \%$ in the surgery group and $6.7 \%$ in the clomiphene group $(\mathrm{p}=0.50)$. While small and likely underpowered, this study further supports the possibility that repair of subclinical varicoceles may have a positive impact on fertility. ${ }^{16}$ Another prospective study randomized 85 men with subclinical varicocele, diagnosed using scrotal thermography, into two groups - high ligation and control (no surgical intervention). ${ }^{17}$ The intervention group demonstrated improved sperm density and TMC at one year post surgery, but no differences in pregnancy rates were observed (6.75\% vs $10.0 \%$; $p>0.50)$. The overall number of pregnancies was small in each group ( $3 v s 4$ ), limiting the ability to draw clear conclusions from these results.

Consistent with other reports, 21 patients in the present study were "downgraded" after varicocele repair. ${ }^{11}$ This likely represents variability between semen analyses, rather than a true decrease in sperm counts secondary to surgery. To further clarify this phenomenon, Samplaski et al. found a higher rate of "decline" in semen parameters in controls than in men having surgery, implying that the decline is not related to the intervention.

The present study has several strengths and limitations that warrant discussion. Although we have fewer subclinical varicoceles than clinical varicoceles, the cohort is relatively large and includes sufficient numbers of patients with palpable and subclinical varicoceles to base our comparisons on. Our analyses are strengthened by post-hoc power analyses demonstrating adequate power $(1-\beta=0.83)$ to detect a difference in total motile count improvement. This work is also strengthened by the clinical translatability of our findings. Using the 'upgrade' in fertility status as used by Cayan et al. and Samplaski et al. allows clinicians to better frame improvements after varicocele repair in a clinically meaningful way. This study is limited in the following ways. First, this is a retrospective chart review and suffers from the limitations inherent with this type of study design. The standard deviation for change in total motile count is large, and likely is due to the variation in starting-point for each patient. For example, a doubling of TMC in a patient with 1 million sperm vs. a patient with 10 million sperm would both be a potentially meaningful improvement, but adds to the variability when reporting changes in TMC. We believe that this data is better understood in the proportion of patients that had a clinically meaningful upgrade rather than the average TMC improvement which must be interpreted with each individual patient in mind. We did not have a nonintervention control group to compare changes in clinical outcomes to outcomes identified in our palpable and subclinical varicocele groups. Our study shares a common weakness in studies evaluating the effect of varicocele repair on fertility, which is the lack of pregnancy and live birth outcome data. Unfortunately, only 8 patients had pregnancy data available in the chart, so no meaningful comparisons could be drawn. In addition, our data may be biased in underestimating improvements in TMC after surgery. As most male infertility clinics can anecdotally attest to, couples who achieve a pregnancy after any intervention are less likely to follow up. The patients included in our study were those who had pre and postoperative semen analyses. If those who achieved pregnancies did not return for a visit, our results would be biased, and underestimate the improvements in semen analysis after varicocele repair. Physicians can now give couples facing infertility tangible information 
regarding what method of achieving pregnancy may be likely to succeed, and how that method may change after varicocele repair. Larger, prospective studies with stricter follow up can better determine the true efficacy of subclinical varicocele repair in infertile men.

\section{Conclusions}

Men with subclinical varicoceles identified by US have similar and clinically meaningful improvement in TMC after varicocele repair compared to men with palpable varicoceles. The data presented should prove useful to practitioners and patients. These findings highlight the need to consider expanding current guidelines to consider varicocele repair for infertile men with subclinical varicoceles.

\section{Acknowledgments}

Source of Funding: This work is supported in part by the National Institute of General Medical Sciences of the National Institutes of Health under Award Number T32GM088129, a 2017 Urology Care Foundation Research Scholars Award to AWP and by NIH grants K12 DK0083014, the Multidisciplinary K12 Urologic Research (KURe) Career Development Program awarded to DJL (NT is a K12 Scholar) and R01DK078121 from the National Institute of Kidney and Digestive Diseases to Dolores J Lamb. The content is solely the responsibility of the authors and does not necessarily represent the official views of the National Institutes of Health.

\section{REFERENCES}

1. Dubin JM, Greer AB, Kohn TP, et al. Men With Severe Oligospermia Appear to Benefit From Varicocele Repair: A Cost-effectiveness Analysis of Assisted Reproductive Technology. Urology. 2018;111:99-103. [PubMed: 29051002]

2. Kohn TP, Kohn JR, Pastuszak AW. Varicocelectomy before assisted reproductive technology: are outcomes improved? Fertil Steril. 2017;108:385-391. [PubMed: 28865536]

3. Kirby EW, Wiener LE, Rajanahally S, et al. Undergoing varicocele repair before assisted reproduction improves pregnancy rate and live birth rate in azoospermic and oligospermic men with a varicocele: a systematic review and meta-analysis. Fertil Steril. 2016;106:1338-1343. [PubMed: 27526630]

4. Clarke BG. Incidence of varicocele in normal men and among men of different ages. JAMA. 1966;198:1121-1122. [PubMed: 5953394]

5. Clavijo RI, Carrasquillo R, Ramasamy R. Varicoceles: prevalence and pathogenesis in adult men. Fertility and Sterility. 2017;108:364-369. [PubMed: 28865534]

6. Damsgaard J, Joensen UN, Carlsen E, et al. Varicocele Is Associated with Impaired Semen Quality and Reproductive Hormone Levels: A Study of 7035 Healthy Young Men from Six European Countries. European Urology. 2016;70:1019-1029. [PubMed: 27423503]

7. Jarow JP, Sharlip ID, Belker AM, et al. Best practice policies for male infertility. J Urol 2002;167:2138-2144. [PubMed: 11956464]

8. Pierik FH, Vreeburg JT, Stijnen T, et al. Improvement of sperm count and motility after ligation of varicoceles detected with colour Doppler ultrasonography. Int J Androl 1998;21:256-260. [PubMed: 9805240]

9. Kim HJ, Seo JT, Kim KJ, et al. Clinical significance of subclinical varicocelectomy in male infertility: systematic review and meta-analysis. Andrologia. 2016;48:654-661. [PubMed: 26589369]

10. Cayan S, Erdemir F, Ozbey I, et al. Can varicocelectomy significantly change the way couples use assisted reproductive technologies? J Urol 2002;167:1749-1752. [PubMed: 11912402]

11. Samplaski MK, Lo KC, Grober ED, et al. Varicocelectomy to "upgrade" semen quality to allow couples to use less invasive forms of assisted reproductive technology. Fertil Steril. 2017;108:609612. [PubMed: 28911932] 
12. Scherr D, Goldstein M. Comparison of bilateral versus unilateral varicocelectomy in men with palpable bilateral varicoceles. J Urol 1999;162:85-88. [PubMed: 10379746]

13. Steckel J, Dicker AP, Goldstein M. Relationship between varicocele size and response to varicocelectomy. J Urol 1993;149:769-771. [PubMed: 8455240]

14. Cantoro U, Polito M, Muzzonigro G. Reassessing the role of subclinical varicocele in infertile men with impaired semen quality: a prospective study. Urology. 2015;85:826-830. [PubMed: 25817105]

15. Seo JT, Kim KT, Moon MH, et al. The significance of microsurgical varicocelectomy in the treatment of subclinical varicocele. Fertil Steril. 2010;93:1907-1910. [PubMed: 19249033]

16. Unal D, Yeni E, Verit A, et al. Clomiphene citrate versus varicocelectomy in treatment of subclinical varicocele: a prospective randomized study. Int J Urol 2001;8:227-230. [PubMed: 11328423]

17. Yamamoto M, Hibi H, Hirata Y, et al. Effect of varicocelectomy on sperm parameters and pregnancy rate in patients with subclinical varicocele: a randomized prospective controlled study. J Urol 1996;155:1636-1638. [PubMed: 8627841] 
25

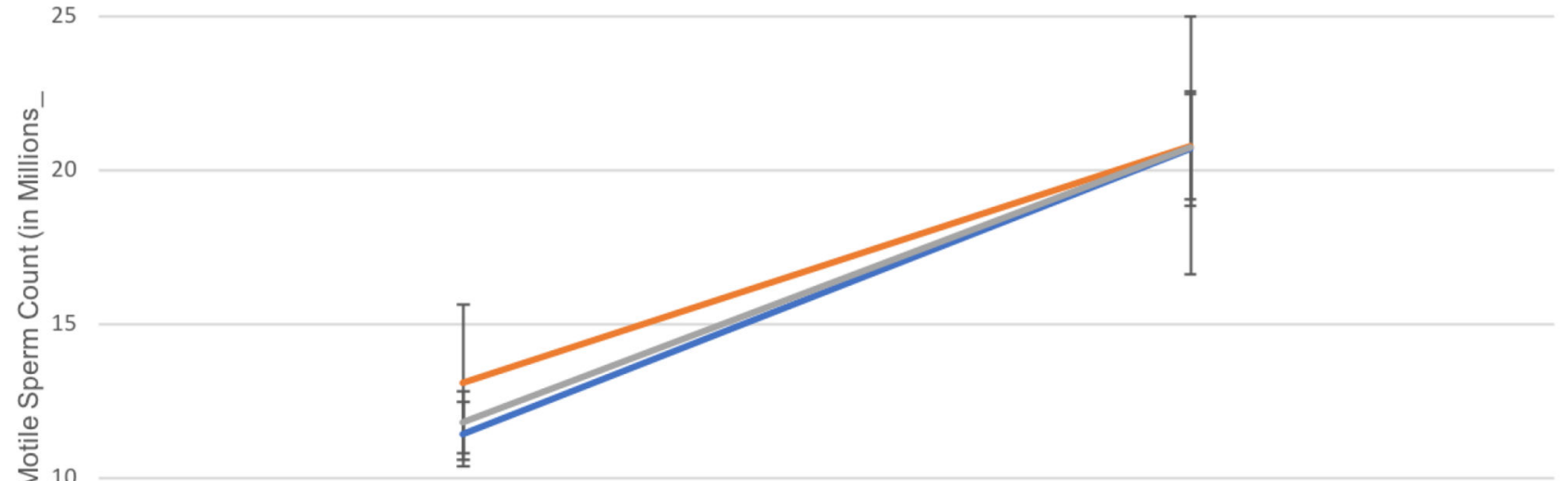

10

욤

5

0

Preoperative

Postoperative

$\longrightarrow$ Clinical $\longrightarrow$ Sub-Clinical $\longrightarrow$ All

Figure 1: Change in Total Motile Count By Type of Varicocele.

Urology. Author manuscript; available in PMC 2019 October 01 
Table 1:

\section{Cohort characteristics}

\begin{tabular}{|l|c|c|c|}
\hline & Palpable (n=144) & Subclinical (n=46) & P value \\
\hline Age (years) & $35.4 \pm 7.0$ & $34.8 \pm 5.9$ & 0.80 \\
\hline Partner Age (years) & $31.5 \pm 4.1$ & $31.3 \pm 3.9$ & 0.74 \\
\hline Duration Trying (months) & $21.7 \pm 19.4$ & $19.7 \pm 24.2$ & 0.65 \\
\hline \% Primary Infertility & $127 / 144(88 \%)$ & $37 / 42(88 \%)$ & 0.92 \\
\hline \% Smokers & $12 / 144(8.3 \%)$ & $4 / 46(8.7 \%)$ & 0.93 \\
\hline BMI & 29.1 & 31.7 & 0.23 \\
\hline \% Bilateral & $106 / 144(74 \%)$ & $33 / 41(80 \%)$ & 0.37 \\
\hline
\end{tabular}


Table 2:

Changes in ART options after varicocele repair for all men. Bolded values represent patients who were upgraded after surgery

\begin{tabular}{|l|l|l|l|l|}
\hline \multicolumn{2}{|c|}{} & \multicolumn{3}{|c|}{ Postoperative } \\
\hline & & \multicolumn{1}{|c|}{ IVF } & \multicolumn{1}{|c|}{ IUI } & \multicolumn{1}{l|}{ Natural } \\
\hline \multirow{4}{*}{ Preoperative } & IVF & $40(49 \%)$ & $\mathbf{1 1}(\mathbf{1 3 \%})$ & $\mathbf{3 1}(\mathbf{3 8 \%})$ \\
\cline { 2 - 5 } & IUI & $4(13 \%)$ & $4(13 \%)$ & $\mathbf{2 2 ( 7 3 \% )}$ \\
\cline { 2 - 5 } & Natural & $9(11 \%)$ & $8(9 \%)$ & $68(80 \%)$ \\
\hline
\end{tabular}



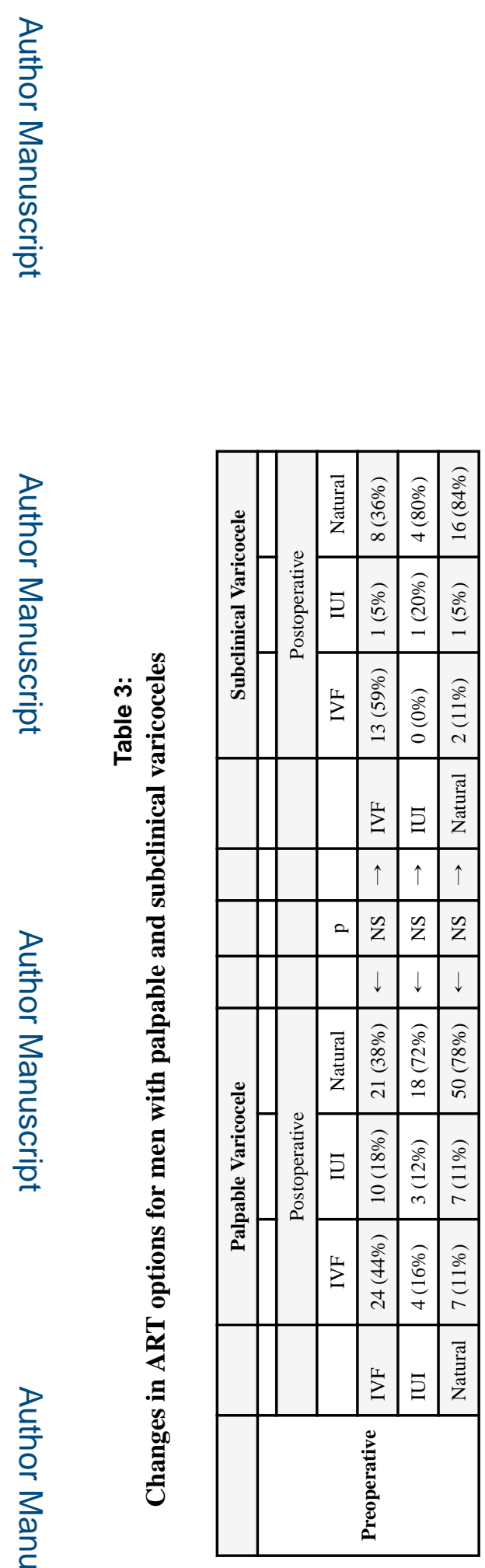

Urology. Author manuscript; available in PMC 2019 October 01. 